\title{
Estilo de aprendizaje y nivel de pensamiento: una apuesta por el rendimiento académico en estudiantes de enfermería de una universidad privada
}

\section{Learning style and cognitive level: A bet on academic performance in nursing students of a private university}

\author{
Sandra Prada-Reyes \\ Jorge Bilbao-Ramírez \\ Gloria Lastre-Amell \\ Janeth Jinete-Acendra \\ Ana Manuel-Ferrer \\ Universidad Metropolitana, Barranquilla, Colombia
}

\section{Resumen}

Objetivo: Relacionar el estilo de aprendizaje, el nivel de pensamiento y el rendimiento académico en los estudiantes de Enfermería de primero a octavo semestre. Método: Estudio cuantitativo, descriptivo y correlacional, de acuerdo con el estilo de aprendizaje propuesto por Kolb, prueba de pensamiento lógico (Tolt) y el promedio de calificaciones por semestre. La muestra fue de 381 estudiantes de I a VIII semestre, a quienes se les aplicó el Test de Comparación Múltiple LSD (Least Significant Difference), se graficaron los intervalos de confianza del rendimiento asociados a cada estilo de aprendizaje. Resultados: Se evidenció que el estilo de aprendizaje Convergente - Asimilador, obtuvo el rendimiento promedio más alto y el estilo Acomodador - Divergente, presentó el rendimiento promedio más bajo. El nivel de pensamiento "Transicional" fue el que obtuvo el rendimiento promedio más alto y el nivel de pensamiento "Concreto" fue el que presentó un rendimiento promedio más bajo. Discusión y Conclusiones: Los estilos de aprendizaje y los niveles de pensamiento si influyen en el rendimiento académico, pero también existe una diversidad de factores que contribuyen al rendimiento académico aceptable de los estudiantes.

Palabras clave: estilos de aprendizaje, pensamiento, rendimiento académico, enfermería.

\begin{abstract}
Objective: The study aims to establish the relationships among learning style, cognitive level, and academic performance among nursing students in their first to fourth years. Method: The paper presents a quantitative, descriptive, and correlational study following the learning style suggested by Kolb, the Test of Logical Thinking, and average grades per semester. The study recruited 381 students who attended semesters I-VIII and who took the least significant difference test. The performance confidence intervals in relation to each learning style were plotted on a graph. Results: The results demonstrated that the converger-assimilator learning style led to the highest average performance, whereas the accommodator-diverger style exhibited the lowest average performance. The transitional cognitive level achieved high levels of average performance, whereas specific cognition led to low levels of average performance. Discussion and Conclusions: Learning styles and cognitive levels influenced academic performance; however, various factors also contributed to the acceptable levels of academic performance among the nursing students.
\end{abstract}

Keywords: learning styles, cognition, academic performance, nursing.
Open Access:

E-ISSN: $2665-2420$ ISSN: $0124-2121$

ARTÍCULO RESULTADO DE INVESTIGACIÓN Copyright $@ 2020$ By Educación y Humanismo

Editor: Dhayana Fernández Matos Universidad Simón Bolívar

Recibido: $17-02-2020$

Aceptado: $30-05-2020$ En línea desde: 09-07-2020 um. 22. 


\section{Introducción}

La presente investigación tuvo como referentes teóricos el modelo sobre el estilo de aprendizaje de Kolb (1975), en las variables nivel de pensamiento y desarrollo cognitivo fundamentadas en la teoría de Jean Piaget (Piaget y Inhelder1973), este enuncia que los estilos de aprendizaje son capacidades de aprender que se acentúan por encima de otras, teniendo en cuenta una predisposición hereditaria como resultado de las experiencias propias y de las exigencias del medio ambiente actual (Gamboa, Garcia y Ahumada 2017). Otra investigación menos antigua del modelo de Kolb (1984), refiere que el modelo se fundamentó en los modelos de aprendizaje definidos por Kurt Lewin, John Dewey y Jean Piaget; esta manera de aprender se apoya en la importancia de la experiencia en los procesos de aprendizaje.

Para Alonso, Gallego y Honey, 1997 (citado por Antelm, Cacheiro y Gil, 2015, p. 1) los estilos de aprendizaje son "los atributos cognitivos, afectivos y fisiológicos, que se utilizan como guías respectivamente estables, de cómo los estudiantes descubren, interaccionan y manifiestan a sus ambientes de aprendizaje".

Los autores Vidal, Canós, Santandreu y Guijarro (2018) en su estudio definieron los cuatros tipos de aprendizaje expresado en la teoría de Kolb (1975), los cuales son los siguientes: Tipo I. Observación reflexiva: Aprenden viendo y escuchando, señalando las ideas y situaciones con una observación meticulosa, gozan intuyendo el significado de las circunstancias y son buenos para comprender sus implicaciones, desde diferentes perspectivas, son individuos, imparciales y muy pensativos, le dan sentido a la experiencia y reflexiona sobre la misma. Tipo II. Conceptualización abstracta: Aprenden pensando. Emplean la lógica e ideas, son buenos para la organización sistemática, análisis cuantitativo, valoran el rigor, la disciplina de las ideas analizadas, la calidad y estética de un sistema conceptual ordenado. Tipo III. Experimentación activa. Aprenden actuando: Se caracterizan por ser muy activos e influidos por la gente y por situaciones cambiantes, aceptan compromisos para lograr objetivos. Intervienen en el entorno que les rodea y les gusta ver resultados. Usan una información nueva para tener una práctica inmediata. Y la Tipo IV. Experiencia concreta. Aprenden sintiendo: Se relacionan bien con los demás. Son buenos para tomar decisiones y trabajan bien en escenarios no estructurados. Aprenden relacionándose con la gente y cuando se envuelven en experiencias reales. Asimilan información ante experiencias directas, innovando, ejerciendo y apreciando.

Así mismo, Kolb (1975) describe los estilos de aprendizaje los cuales son influidos por diferentes trabajos y tareas y pueden cambiar con el desarrollo de la persona y el entorno que le rodea, siendo esto denominados: Convergente (Experimentación actival conceptualización abstracta). Las personas se caracterizan por resolver problemas, tomar decisiones, y aplicar estas ideas a la práctica, reciben este calificativo porque trabajan 
mejor en ambientes donde hay una única respuesta correcta y una solución a un problema. Divergente (experiencia concreta/ observación reflexiva) tienen destreza imaginativa y cognición del significado y valores. Aplican más la observación que la acción. Asimilador (observación reflexiva / conceptualización abstracta) se caracteriza por su razonamiento de lo particular a lo general. Estudian con ideas abstractas y conceptos, instituyendo patrones conceptuales, experimentando, resolviendo problemas, pensando alternativas de soluciones, analizando, reflexionando, teorizando información cuantificada y actividades estructuradas. Y el Acomodador (Experiencia concreta / experimentación activa) aprenden mediante experiencias, planeando tareas y teniendo nuevos conocimientos (Piorno-Ruiz, 2014). Medina y Medina (s. f. citado por Salas Cabrera, 2014), expresa que los estilos de aprendizaje reconocen al profesor como facilitador del aprendizaje, además tiene la eventualidad de disponer su estilo de enseñanza con la forma de instituir del grupo al que enseña.

Investigaciones anteriores han mostrado que parece existir una relación entre la capacidad intelectual y los estilos de aprendizaje. En una investigación sobre estilos de aprendizaje e inteligencia en estudiantes universitarios de Arequipa, Perú se pudo comprobar que "los estudiantes con menor capacidad intelectual presentan estilos de aprendizaje prácticos, mientras que los estudiantes con mayor capacidad intelectual tienen un estilo de aprendizaje divergente, asimilador y convergente" (Arias, 2014, p. 99).

Por otro lado, en cuanto a los niveles de pensamiento, Jean Piaget uno de los pensadores más influyentes en la Psicopedagogía y cuyos postulados siguen vigentes, señala la importancia de identificar la capacidad del estudiante para trabajar en el proceso de construcción del conocimiento a partir del pensamiento formal (Soriano G., 2010).

El pensamiento formal se desarrolla de manera espontánea en todos los adolescentes y adultos, cuando este se consolida en una persona, se manifiesta la madurez cognitiva permitiéndole usarlo como un recurso efectivo sobre los diversos problemas, haciéndose vital para la comprensión de la ciencia (Labinowiwics, 1982).

En la etapa de operaciones formales y a lo largo de este periodo se desarrolla un pensamiento abstracto, formal y lógico; el procesamiento ya no está ligado a sucesos que los individuos observan en el entorno si no que son capaces de hacer abstracciones y resolver problemas.La teoría piagetiana refiere que el pensamiento formal aparece en la adolescencia, sin embargo, diversas investigaciones realizadas en países desarrollados o en vía de desarrollo, demuestran que los jóvenes escolarizados no manejan esquemas de pensamiento formal (Jaramillo y Puga, 2016).

El desarrollo de las habilidades de pensamiento ha sido un tema de especial interés para científicos y educadores en las últimas décadas. En los 70 surge la inquietud ya que se observa en jóvenes universitarios de muchas instituciones educativas, alteraciones en el desempeño intelectual y las causas no están claramente establecidas (Amestoy, 2002). 
Así mismo, en un estudio sobre niveles de pensamiento y resolución de problemas matemáticos por parte de estudiantes de primero a octavo semestre del programa de Psicología de una universidad pública de Santa Marta (Magdalena), se encontró que un $56.7 \%$ tuvo pensamiento concreto, $37.4 \%$ en transición y el $5.8 \%$ formal; los resultados señalan la existencia niveles de pensamiento en la resolución de problemas matemáticos (Quintero, Suarez, García y Vanegas 2012).

Distintos autores (Cózar et al., 2016; Rodríguez 2016; Gamboa, Briceño, y Camacho 2015; Arias, Zegarra \& Velarde 2014) en sus investigaciones han manifestado que existen distintos modelos teóricos que especifican y clasifican los estilos de aprendizaje, no obstante, han seleccionado el modelo de Kolb por su capacidad para la sucesión de aprendizajes que enlaza la actuación, la reflexión, la teorización y la experimentación.

En Colombia, el Ministerio de Educación Nacional, (2009) mediante el Decreto $N^{\circ}$ 1290/2009, reglamenta la evaluación del aprendizaje de los estudiantes mediante la identificación de los estilos de aprendizaje, características personales, intereses, ritmos de desarrollo, con el propósito de valorar sus avances durante el proceso de enseñanzaaprendizaje (Ministerio de Educación, Colombia, 1993). Es por ello que hablar de estilos de aprendizaje: se relaciona con el hecho de que cada persona utiliza su propio método o estrategia a la hora de aprender (Saavedra y Parra, 2014).

Es así que la necesidad de revelar el rendimiento académico como un fenómeno complejo no está solo en orientar los procesos de clasificación, sino en el conocimiento del estudiante que vive la clasificación desde diferentes perspectivas, destacando entre ellas la importancia de identificar el estilo de aprendizaje, nivel de pensamiento y su relación con el rendimiento académico en la labor diaria como profesor, con el objeto de establecer estrategias de reflexión, intervención y transformación, logrando así cambios significativos en el rendimiento académico de los educandos. Este tema es de gran interés ya que, además de otros factores, el bajo rendimiento académico es un problema usual presente en diferentes instituciones públicas y privadas de Colombia y en los diferentes niveles de educación, situación que afecta de manera significativa la culminación de los procesos académicos (Rico-Hernández, 2016).

Cuando los dicentes inician estudios de educación superior, se espera que tengan desarrollado unos hábitos de estudios y un estilo de aprendizaje instaurado fruto de unas estrategias que le deben acarrear al éxito académico (López-García, 1996), sin embargo, en la labor diaria como docentes del programa de Enfermería, encontramos casos de estudiantes que, si bien en su rendimiento académico alcanzan promedios altos, muestran dificultades en la realización de actividades académicas que involucran procesos cognitivos complejos. De acuerdo con informes del Departamento de Bienestar Universitario sobre las causas que pueden contribuir al abandono de los estudios y al fracaso estudiantil en el programa de Enfermería de una universidad privada de Barranquilla, se evidencia que la primera causa la constituye el factor económico $(37,3 \%)$, seguida por las dificultades 
académicas (36\%) y personales $(16,8 \%)$, un $19,7 \%$ representan las causas no determinadas a la fecha (Universidad Metropolitana, 2015).Como se pudo observar, las dificultades académicas entre ellas el bajo rendimiento académico, ocupan el segundo lugar, situación que motiva al grupo investigador a identificar los factores presentes en dicha problemática y esto a su vez nos motiva a emprender la tarea de buscar la calidad educativa mediante la identificación del estilo de aprendizaje, nivel de pensamiento y su relación con el rendimiento académico en los estudiantes del programa de Enfermería de primero a octavo semestre en una Institución de Educación Superior en Barranquilla. En esta línea es importante destacar lo señalado por Garizabalo (2012):

Muchos de los docentes al iniciar el curso a su cargo no incluyen dentro de sus diagnósticos de entrada, un estudio sobre los diferentes estilos de aprender que poseen sus estudiantes; esto se puede deber a un desconocimiento por parte de ellos de este factor que tanta importancia e influencia tiene en el rendimiento académico (p.20)

Los nuevos desafíos a los que se ven enfrentados los docentes exigen que se establezcan estrategias también novedosas, incluso se puede hablar de un desaprendizaje de las acostumbradas y requiere reflexionar en torno a la educación universitaria, teniendo en cuenta los estilos de aprendizaje y niveles de pensamiento de los estudiantes involucrados en la experiencia de aprender. En este contexto, el objetivo de esta investigación es relacionar el estilo de aprendizaje, nivel de pensamiento y el rendimiento académico delos estudiantes de Enfermería de una universidad privada de Barranquilla.

\section{Método}

Fue un estudio cuantitativo, descriptivo y correlacional; la población objeto de estudio estuvo conformada por estudiantes de I a VIII semestre del programa de Enfermería, abordados como un estudio poblacional, por tanto, no se calculó tamaño de muestra (Ver tabla 1). Los criterios de inclusión que se tomaron en cuenta fueron: 1) que los estudiantes estuvieran matriculados en el Programa de Enfermería de una universidad privada de Barranquilla y 2) que estuvieran cursando de primero a octavo semestre.

Tabla 1.

Calculó tamaño de muestra de estudiantes de I a VIII semestre del programa de Enfermería

\begin{tabular}{cccc}
\hline SEMESTRE & $\begin{array}{c}\text { No ESTUDIANTES } \\
\text { MATRICULADOS }\end{array}$ & $\begin{array}{c}\text { ESTUDIANTE } \\
\text { ENCUESTADOS }\end{array}$ & $\begin{array}{c}\text { PORCENTAJE DE } \\
\text { ENCUESTADOS }\end{array}$ \\
\hline I & 58 & 46 & $79.3 \%$ \\
\hline II & 99 & 63 & $63.6 \%$ \\
\hline III & 48 & 30 & $62.5 \%$ \\
\hline IV & 72 & 44 & $61.1 \%$ \\
\hline V & 71 & 57 & $80.2 \%$ \\
\hline VI & 80 & 62 & $63.8 \%$ \\
\hline VII & 72 & 46 & $50 \%$ \\
\hline VIII & 66 & 33 &
\end{tabular}




Total $\quad 560 \quad 381 \quad 68.03 \%$

Fuente: Listado de estudiantes de Enfermería matriculados. Elaboración propia (2020).

Para la recolección de la información se aplicó el inventario de estilos de aprendizaje de Kolb (1975), instrumento que estuvo constituido por nueve conjuntos de cuatro palabras cada uno, y el investigador que lo está aplicando debe manifestar de manera sistemática en cada conjunto (en una escala del 1 al 4, en donde 4 es la que mejor define su estilo, 3 en menor medida, 2 en menor grado, y un 1 a la menos) las palabras que mejor describen el estilo de aprendizaje. Posteriormente se realizó una sumatoria específica de todas las escalas, identificando y relacionando las respuestas con alguno de las cuatro orientaciones de estilos de aprendizaje: experiencia concreta, observación reflexiva, conceptualización abstracta, y experimentación activa.

Para saber los estados de los niveles de pensamiento en los educandos se aplicó la Prueba de Pensamiento Lógico (TOLT), diseñado por Tobin y Capie (1981):

Es una prueba de razonamiento lógico que determina los niveles de pensamiento a través del número de respuestas correctas así: el nivel concreto (0-3), transicional (4-6) y formal (710). Esta prueba evaluó los niveles de razonamiento proporcional, control de variable, de razonamiento probabilístico y razonamiento combinatorio, características propias del pensamiento formal. Su validez convergente es de 0.80 y la confiabilidad es de 0.73 , utilizando el Coeficiente de Kuder Richardson (p. 25)

Para la variable rendimiento académico se utilizó el Registro Académico de los estudiantes que hicieron parte de la población en estudio, al cual se accedió, previa autorización del Departamento de Admisiones y registro, así como del consentimiento informado o asentimiennto de los estudiantes participantes. El rendimiento académico se logró del promedio acumulado de notas de acuerdo al semestre cursado por los educandos de Enfermería del primero a octavo semestre durante el II periodo del año 2015 y se estableció la escala evaluación según la siguiente operacionalización: Insuficiente 1.0- 1.99; Deficiente 2.0-2.99; Aceptable 3.0-3.99, Bueno 4.0- 4.49; Excelente 4.5- 5.0.

Para establecer el grado de relación entre las variables, estilo de aprendizaje, niveles de pensamiento y rendimiento académico se aplicó el Test de Comparación Múltiple Least Significant Difference (LSD) de Fisher, utilizando el ANOVA para crear intervalos de confianza para todas las diferencias de variables entre las medias de los niveles de los factores, controlando al mismo tiempo la tasa de error individual en un nivel especificado, y después se graficaron los intervalos de confianza de los rendimientos promedios asociados a cada estilo de aprendizaje, coeficiente de correlación de Pearson. 
Así mismo se salvaguardaron las consideraciones éticas que avalaron el rigor ético, científico y metodológico del estudio. Es decir, los criterios constituidos en la Resolución 008430 de (1993), las pautas del Comité de Organizaciones Internacionales de las Ciencias Médicas (Consejo de Organizaciones Internacionales de las Ciencias Médicas CIOMS-OMS, 2016), y los principios éticos establecidos en la Ley 911 de 2004 (Ministerio de Educación Nacional, 2004). Se presentó a los estudiantes el consentimiento informado en donde autorizaron su colaboración en la investigación, se procedió a la entrega de los instrumentos, luego de ser examinados, se sistematizaron en la base de datos SPSS Versión 22. Subsiguientemente se aplicaron datos estadísticos correspondientes con los objetivos de investigación.

\section{Resultados}

\section{Estilos de aprendizaje y rendimiento académico}

Para determinar si el estilo de aprendizaje presentó algún tipo de relación con el rendimiento académico de los estudiantes, se evidenció que el Estilo de Aprendizaje "Convergente - Asimilador" es el que obtiene el rendimiento promedio más alto y el estilo de aprendizaje Acomodador - Divergente fue el que presentó rendimiento promedio más bajo entre los Estilos de Aprendizaje (Figura 1).

Figura 1.

Estilos de aprendizaje y Rendimiento Académico

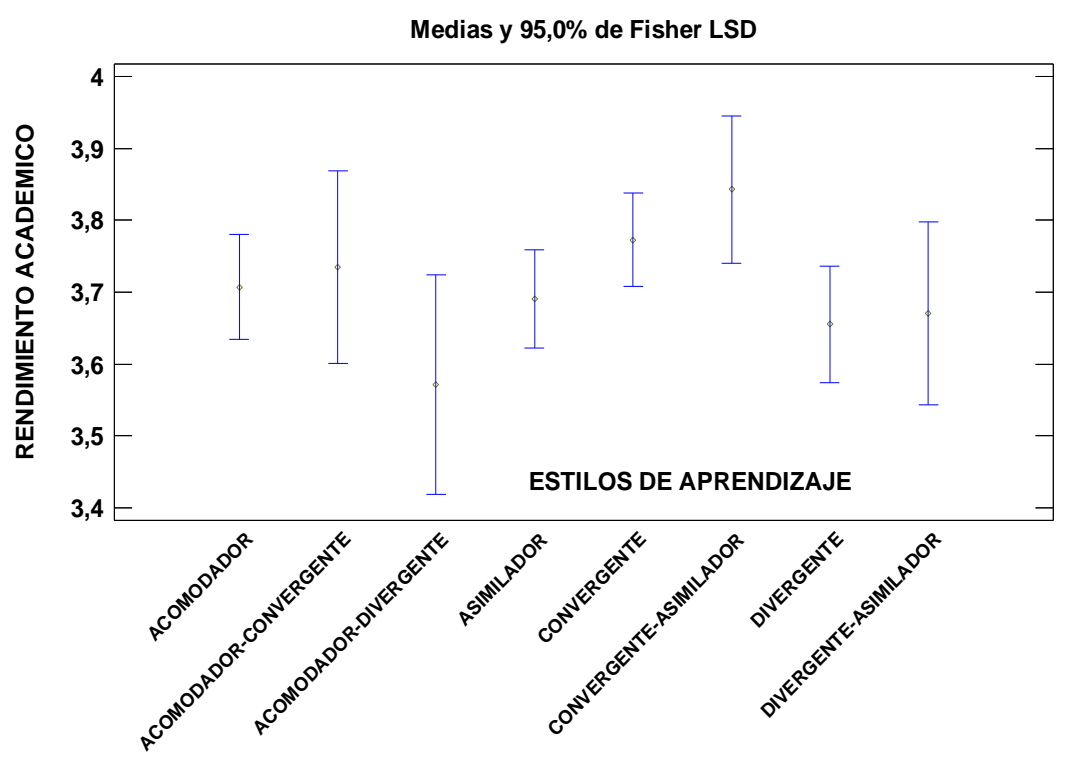

Fuente: Elaboración propia (2020) 


\section{Niveles de pensamiento y rendimiento académico}

Se evidenció que el nivel de pensamiento "Transicional" es el que obtiene el rendimiento promedio más alto y el nivel de pensamiento "Concreto" fue el que presentó rendimiento promedio más bajo entre los distintos Niveles de Pensamiento (Figura 2)

\section{Figura 2.}

Niveles de pensamiento y rendimiento académico

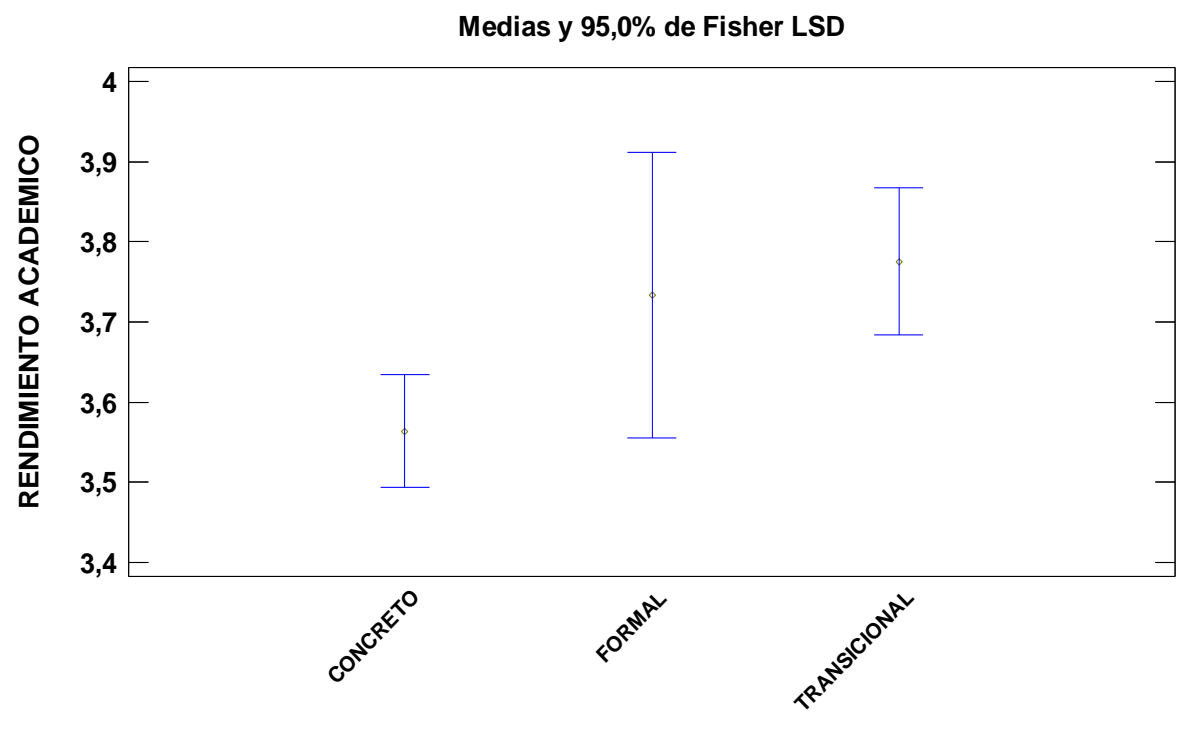

NIVELES DE PENSAMIENTO

Fuente: Elaboración propia (2020)

\section{Análisis estadístico para el Nivel de Pensamiento}

Dado que el valor $\mathrm{p}$ de la prueba de Análisis de Varianza (ANOVA) resulto menor de 0,05 se concluye que las calificaciones promedio de los estudiantes clasificados según el estilo de aprendizaje presentan diferencias estadísticamente significativas.

Para determinar qué diferencias significativas que se dieron entre los niveles de pensamiento se aplicó la prueba LSD de Fisher la cual arrojó las siguientes diferencias (Figura 3). 
Figura 3.

Análisis estadístico para el Nivel de Pensamiento

Prueba LSD de Fisher usando una confianza del 95\%

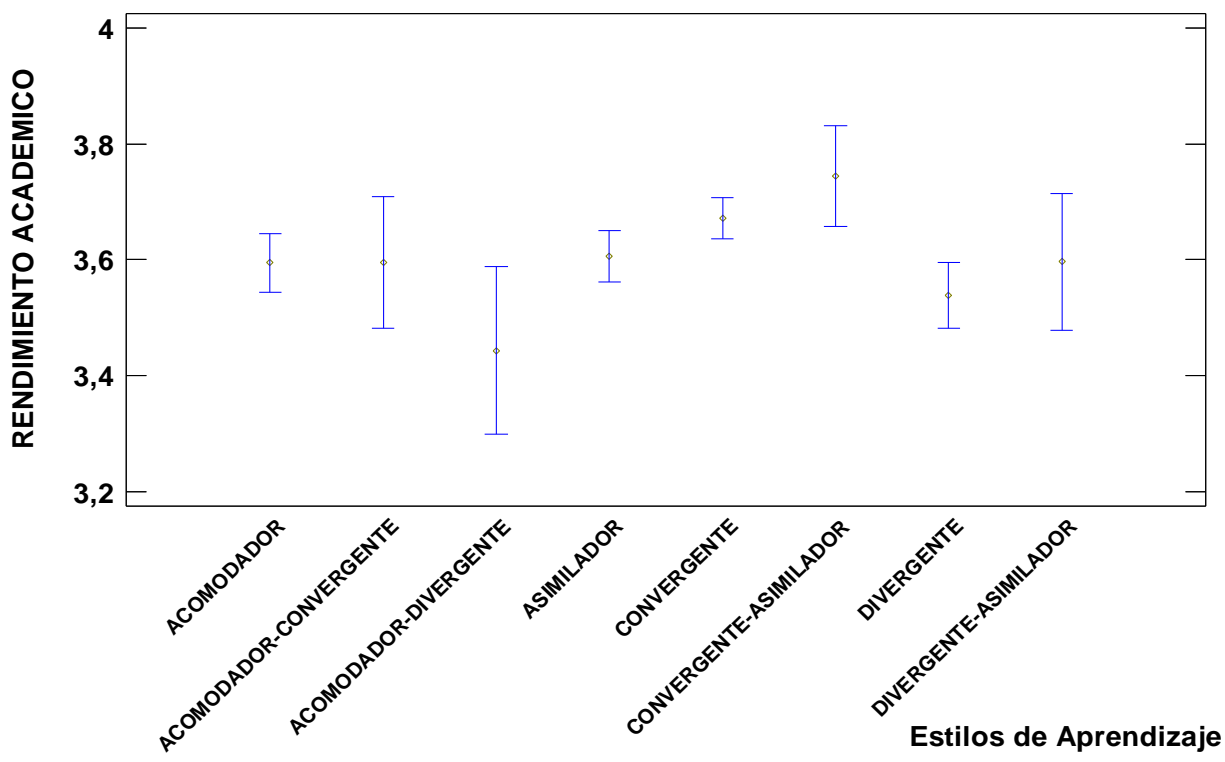

Fuente: Elaboración propia (2020)

\section{Rendimiento académico/Estilos de aprendizaje/ Niveles de pensamiento}

Se evidencia que los estudiantes que fueron clasificados como Asimilador/Concreto presentaron el más alto porcentaje catalogados como "Buenos" en su rendimiento académico, representando el 2,62\% del total de estudiantes. Con respecto a la clasificación de "Deficientes", los estudiantes clasificados como Asimilador/Concreto fueron los que más obtuvieron este desempeño académico, fueron el 1,31\% del total de estudiantes (Tabla 2).

Tabla 2.

Rendimiento académico /estilos de aprendizaje/niveles de pensamiento

\begin{tabular}{ccccc}
\hline Suma de ACUMULADO & $\begin{array}{c}\text { Etiquetas de } \\
\text { columna }\end{array}$ & & & \\
\hline ETIQUETAS DE FILA & ACEPTABLE & BUENO & DEFICIENTE & $\begin{array}{c}\text { Total } \\
\text { general }\end{array}$ \\
\hline Acomodador & $94,4 \%$ & $1,8 \%$ & $3,8 \%$ & $100 \%$ \\
\hline Concreto & $93,9 \%$ & $2 \%$ & $4,1 \%$ & $100 \%$ \\
\hline Formal & $100 \%$ & $0 \%$ & $0 \%$ & $100 \%$ \\
\hline Transicional & $100 \%$ & $0 \%$ & $0 \%$ & $100 \%$ \\
\hline Acomodador-Convergente & $100 \%$ & $0 \%$ & $0 \%$ & $100 \%$ \\
\hline Concreto & $100 \%$ & $0 \%$ & $0 \%$ & $100 \%$ \\
\hline Acomodador-Divergente & $90 \%$ & $0 \%$ & $10 \%$ & $100 \%$ \\
\hline
\end{tabular}




\begin{tabular}{ccccc}
\hline Concreto & $90 \%$ & $0 \%$ & $10 \%$ & $100 \%$ \\
\hline Asimilador & $76,5 \%$ & $19 \%$ & $4,5 \%$ & $100 \%$ \\
\hline Concreto & $77,4 \%$ & $17 \%$ & $5,6 \%$ & $100 \%$ \\
\hline Formal & $100 \%$ & $0 \%$ & $0 \%$ & $100 \%$ \\
\hline Transicional & $69,1 \%$ & $30,9 \%$ & $0 \%$ & $100 \%$ \\
\hline Convergente & $94,3 \%$ & $5,1 \%$ & $0,6 \%$ & $100 \%$ \\
\hline Concreto & $96,4 \%$ & $2,9 \%$ & $0,7 \%$ & $100 \%$ \\
\hline Formal & $100,0 \%$ & $0 \%$ & $0 \%$ & $100 \%$ \\
\hline Transicional & $79,3 \%$ & $20,7 \%$ & $0 \%$ & $100 \%$ \\
\hline Convergente-Asimilador & $84,9 \%$ & $15,1 \%$ & $0 \%$ & $100 \%$ \\
\hline Concreto & $94,2 \%$ & $5,8 \%$ & $0 \%$ & $100 \%$ \\
\hline Transicional & $30,1 \%$ & $69,9 \%$ & $0 \%$ & $100 \%$ \\
\hline Divergente & $86,4 \%$ & $9,0 \%$ & $4,6 \%$ & $100 \%$ \\
\hline Concreto & $87,0 \%$ & $7,7 \%$ & $5,3 \%$ & $100 \%$ \\
\hline Transicional & $81,7 \%$ & $18,3 \%$ & $0 \%$ & $100 \%$ \\
\hline Divergente-Asimilador & $100 \%$ & $0 \%$ & $0 \%$ & $100 \%$ \\
\hline Concreto & $100 \%$ & $0 \%$ & $0 \%$ & $100 \%$ \\
\hline Transicional & $100 \%$ & $0 \%$ & $0 \%$ & $100 \%$ \\
\hline Total general & $89,2 \%$ & $8,2 \%$ & $2,6 \%$ & $100 \%$ \\
\hline
\end{tabular}

Fuente: Elaboración propia (2020)

\section{Análisis estadístico para el estilo de aprendizaje}

Con relación a la clasificación como "Aceptables" en su rendimiento académico, los estudiantes clasificados como Convergente/Concreto fueron los que más aportaron a esta clasificación del rendimiento con $28,08 \%$ del total de estudiantes clasificados (Tabla3).

Tabla 3.

Análisis estadístico para el Estilo de aprendizaje ANOVA PARA RENDIMIENTO ACADEMICO POR ESTILOS DE APRENDIZAJE

\begin{tabular}{lccccc}
\hline \multicolumn{1}{c}{ Fuente } & $\begin{array}{c}\text { Suma de } \\
\text { Cuadrados }\end{array}$ & GI & $\begin{array}{c}\text { Cuadrado } \\
\text { Medio }\end{array}$ & Razón-F & Valor-P \\
\hline Entre grupos & 1,34828 & 7 & 0,192611 & 2,24 & 0,0308 \\
\hline Intra grupos & 32,6514 & 379 & 0,0861514 & & \\
\hline Total (Corr.) & 33,9997 & 386 & & & \\
\hline
\end{tabular}

Fuente: Elaboración propia (2020). 


\section{Rendimiento académico por niveles de pensamiento}

Dado que el valor $p$ de la prueba de Análisis de Varianza (ANOVA) resulto menor de 0,05 se concluye que las calificaciones promedio de los estudiantes clasificados según el nivel de pensamiento presentan diferencias estadísticamente significativas (Tabla 4).

Tabla 4

ANOVA PARA RENDIMIENTO ACADEMICO POR NIVELES DE PENSAMIENTO

\begin{tabular}{cccccc}
\hline Fuente & $\begin{array}{c}\text { Suma de } \\
\text { Cuadrados }\end{array}$ & $\boldsymbol{G l}$ & $\begin{array}{c}\text { Cuadrado } \\
\text { Medio }\end{array}$ & Razón-F & Valor-P \\
\hline Entre grupos & 1,96841 & 2 & 0,984203 & 11,80 & 0,0000 \\
\hline Intra grupos & 32,0313 & 384 & 0,0834148 & & \\
\hline Total (Corr.) & 33,9997 & 386 & & & \\
\hline
\end{tabular}

Fuente: Elaboración propia (2020).

Figura 4.

Rendimiento académico por niveles de pensamiento

Prueba LSD de Fisher usando una confianza del $95 \%$

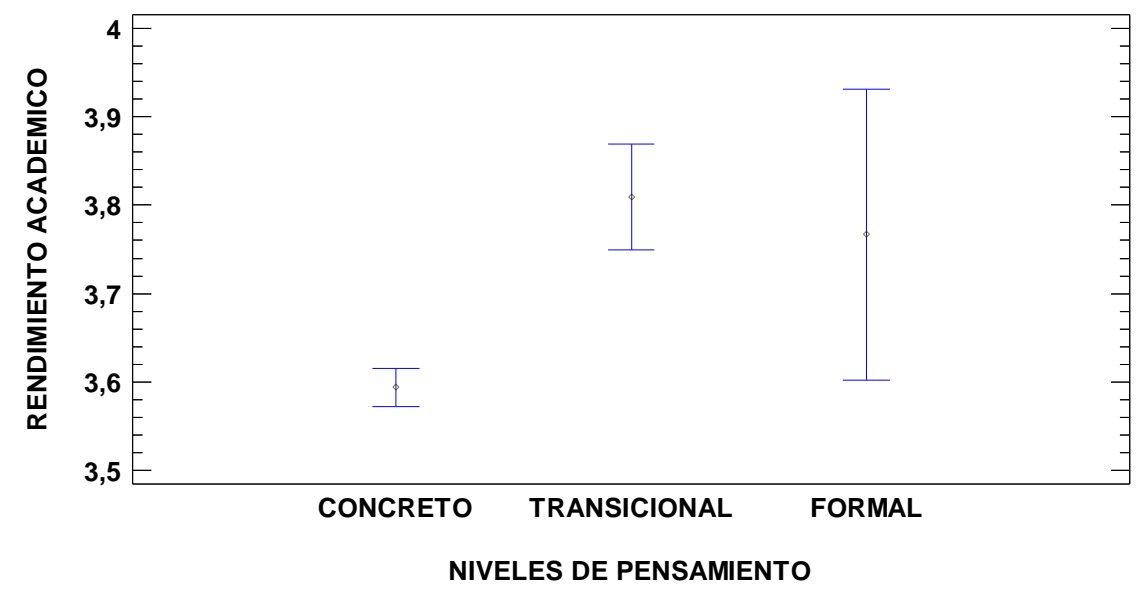

Fuente: Elaboración propia (2020). 


\section{Discusión}

En la investigación de los estilos de aprendizaje en estudiantes de Enfermería de una universidad privada de Barranquilla se identificó que el estilo de aprendizaje más frecuente fue el convergente con el 34.6\%, resultado similar con el estudio de Sánchez y Alejos (2019); Barría, Igor y Marín (2019). El estudio arrojó que el estilo acomodador divergente se presentó con menor frecuencia (2.1\%), y el $86.61 \%$ de ellos se encuentran en el nivel de pensamiento concreto; el $1.5 \%$ tiene pensamiento transicional y el 1,57\% pensamiento formal. Por lo cual, se puede concluir que el mayor porcentaje de los estudiantes que conforma la población objeto de estudio aún no posee un pensamiento formal; así mismo, en las investigaciones como la de Iriarte, Cantillo y Polo (2000) se encontró que aparición tardía del pensamiento formal, y el nivel de pensamiento fue concreto representó la mayoría.

El nivel de pensamiento "transicional" fue el que obtuvo el rendimiento promedio más alto y el nivel de pensamiento "concreto" fue el que presentó un rendimiento académico aceptable, entre los niveles de pensamiento. De igual forma, lo indica el estudio realizado por Cerchiaro, Paba, Tapia y Sánchez (2006) quienes señalan que el nivel de pensamiento

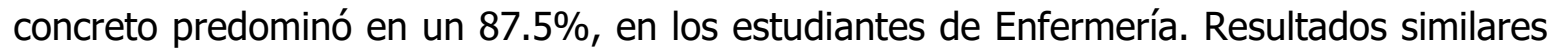
a los datos encontrados en la investigación de Quintero, et al. (2012), siendo el nivel de pensamiento concreto predominante en un $56,7 \%$.

Así mismo, se identificó que el estilo de aprendizaje acomodador divergente y el nivel de pensamiento concreto, prevalece en los estudiantes con un rendimiento académico aceptable. De acuerdo a las características descritas por Kolb (1975), en el estilo de aprendizaje convergente sus fortalezas son la definición y resolución de problemas (Gómez Pawelek, 2003). Y el estudio de Rivera, Pirro, Vargas, Bollet y Flores (2017) encontró que los estilos de aprendizaje de los estudiantes encuestados la mayoría tuvo preferencia por el estilo de aprendizaje acomodador, es decir, prefieren aprender de manera directa, pragmática, sin un minucioso análisis lógico. Además, se puede concluir que el rendimiento académico es un constructo multifactorial y debe orientarse desde una perspectiva holística.

\section{Conclusiones}

Se concluye que al relacionar el estilo de aprendizaje, el nivel de pensamiento y el rendimiento académico en los estudiantes de Enfermería de primero a octavo semestre se evidenció que de las características principales de los cuatro tipos de estilos de aprendizaje según Kolb, en un mayor porcentaje fue el Convergente, es decir que los estudiantes están en capacidad para resolver problemas, tomar decisiones, y aplicaciones 
prácticas de ideas, sin dejar de mencionar el estilo acomodador y divergente; y en relación al nivel de pensamiento fue el concreto, debido a que se centra en un pensamiento literal en un mundo físico y sus elementos. Igualmente, se identificó que el rendimiento académico fue aceptable.

Considerando que los estilos de aprendizaje abren camino a que la experiencia de aprendizaje para el profesor como para el dicente sea significativamente más personalizada y consciente del dinamismo en el que se cimienta el proceso de aprendizaje. Además, los estilos de aprendizaje y los niveles de pensamiento si influyen en el rendimiento académico, pero también existe una diversidad de factores que contribuyen al rendimiento académico aceptables de los estudiantes.

Por lo cual se debe sensibilizar el cuerpo de profesores sobre la importancia de identificar los estilos de aprendizaje, nivel de pensamiento de sus educandos con el objeto de fortalecer los métodos de enseñanza- aprendizaje. Y proponer a los docentes la importancia de constituir equipos de trabajo con estudiantes de diferente estilo de aprendizaje capaces de tener conciencia de las diferencias entre sí mismos y los demás.

\section{Referencias}

Amestoy, M. (2002). La investigación sobre el desarrollo y la enseñanza de las habilidades de pensamiento. Revista electrónica de investigación educativa, 4(1), 01-32. http://www.scielo.org.mx/scielo.php?script=sci_arttext\&pid=S160740412002000100010\&lng=es\&tlng=es.

Antelm, AM., Cacheiro, ML., y Gil, AJ. (2015). Análisis del fracaso escolar desde la perspectiva del alumnado y su relación con el estilo de aprendizaje. Educación y Educadores, 18(3), 471-489. https://www.redalyc.org/pdf/834/83443150006.pdf

Arias, W L, Zegarra, J, \& Justo, O. (2014). Learning and metacognition styles in psychology students from Arequipa. Liberabit, 20(2), 267-279. http://www.scielo.org.pe/pdf/liber/v20n2/a08v20n2.pdf

Arias, W. (2014). Estilos de aprendizaje e inteligencia en estudiantes universitarios de Arequipa, Perú. Revista Journal of Learning Styles. 7(14), 88-107. http://revistaestilosdeaprendizaje.com/article/view/996

Barría, L., Igor, Y., y Marín, C. (2019).Estilo de aprendizaje predominante en estudiantes de $1^{\circ}$ a $5^{\circ}$ año de la carrera de enfermería de Universidad Santo Tomas. REIDU. 1(1): 148-173. http://revistas.uantof.cl/index.php/REIDU/article/view/12 
Cerchiaro, E., Paba, C., Tapia, E., y Sánchez, L. (2006). Nivel de pensamiento, rasgos de personalidad promedios academicos en estudiantes universitarios. Revista Duazary, 3(1):81-89.

https://revistas.unimagdalena.edu.co/index.php/duazary/article/view/611

Consejo de Organizaciones Internacionales de las Ciencias Médicas-Organización Mundial de la Salud. (2016). Pautas éticas internacionales para la investigación biomédica en seres humanos. https://cioms.ch/wp-content/uploads/2017/12/CIOMSEthicalGuideline_SP_INTERIOR-FINAL.pdf

Cózar-Gutiérrez, R., De Moya-Martínez, M V., Hernández-Bravo, J A., y Hernández-Bravo, J R. (2016). Conocimiento y Uso de las Tecnologías de la Información y las Comunicaciones (TIC) según el Estilo de Aprendizaje de los Futuros Maestros. Formación universitaria, 9(6), 105-118. http://dx.doi.org/10.4067/S071850062016000600010

Gamboa, M. C., García, Y., y Ahumada De La Rosa, V. (2017). Estilos de aprendizaje. Libros Universidad Nacional Abierta Y a Distancia, (2017), 66-91. https://hemeroteca.unad.edu.co/index.php/book/article/view/2484

Gamboa, M., Briceño, J., y Camacho, J P. (2015). Caracterización de estilos de aprendizaje y canales de percepción de estudiantes universitarios. Opción, 31(3), 509-527. https://www.redalyc.org/pdf/310/31045567026.pdf

Garizabalo, C. (2012). Estilos de aprendizaje en estudiantes de enfermería y su relación con el desempeño en las pruebas saber pro. Journal of Learning Styles, 5(9), 97110. http://revistaestilosdeaprendizaje.com/article/view/950

Gómez, J. (2003). El aprendizaje experiencial. https://www.ecominga.uqam.ca/PDF/BIBLIOGRAPHIE/GUIDE_LECTURE_5/1/3.G omez_Pawelek.pdf

Iriarte, F., Cantillo, K., y Polo A. (2000). Relación entre el Nivel dePensamiento y el estilo cognitivo dependencia-independencia de Campo en estudiantes universitarios.

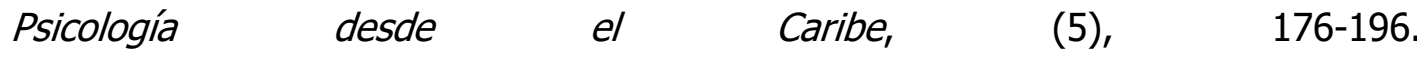
http://rcientificas.uninorte.edu.co/index.php/psicologia/article/viewFile/761/9455

Jaramillo, LM., y Puga Peña, LA. (2016). El pensamiento lógico-abstracto como sustento para potenciar los procesos cognitivos en la educación. Sophia, colección de Filosofía de la Educación, 21(2): 31-55. https://www.redalyc.org/pdf/4418/441849209001.pdf

Kolb, D., y Fry, R. (1975). Toward an applied theory of experiential learning. In C. Cooper (Ed.) Theories of Group Processes. N.Y: John Wiley y Sons. 
Kolb, D. A. (1984). Experiential learning: experience as the source of learning and development. New Jersey: Prentice Hall.

Labinowicz, E. (1982). Introducción a Piaget: Pensamiento, Aprendizaje, Enseñanza. http://www.imetyd.org.mx/archivos/ARCH\%20DOMINICAL\%202013/PIAGET/Piage t\%20biografia\%201.pdfhttp://presencias.net/indpdm.html?http://presencias.net/ed ucar/ht1026.html

López-García, JM. (1996). Los estilos de aprendizaje y los estilos de enseñanza. Un modelo de categorización de estilos de aprendizaje de los alumnos de enseñanza secundaria desde el punto de vista del profesor. Anales de Psicología, 12(2), 179184.

Ministerio de Educación Nacional. (2009, 16 de abril). Decreto $N^{\circ} 1290$ de 2009. Evaluación del aprendizaje y promoción de los estudiantes de los niveles de educación básica y media. https://www.mineducacion.gov.co/1621/articles187765_archivo_pdf_decreto_1290.pdf

Ministerio de Educación Nacional. (19934, 4 de octubre). Resolución 008430 de 1993. https://www.minsalud.gov.co/sites/rid/Lists/BibliotecaDigital/RIDE/DE/DIJ/RESOLU CION-8430-DE-1993.PDF

Ministerio de Educación Nacional. (2004, 5 de octubre). Ley 911 del 2004. Congreso de Colombia. Diario $\quad$ oficial $\quad \mathrm{n}^{\circ}$. 45.693. http://www.secretariasenado.gov.co/senado/basedoc/ley_0911_2004.html

Piaget, J., y Inhelder, B. (1973). Psicología del niño. España: Morata.

Piorno-Ruiz, Y. (2014). Estilo de aprendizaje predominante en los estudiantes del segundo año de Ingeniería Forestal de la Universidad de Guantánamo. EduSol, 14(49), 1-8.

Quintero, L., Suarez, Y., García, G., y Vanegas, J. (2012). Niveles de pensamiento y resolución de problemas matemáticos en los estudiantes del programa psicología de una universidad pública de Santa Marta (Magdalena). Revista Internacional de $\begin{array}{llll}\text { Ciencias de la Salud: Duazary, 9(2), 123-131. } & \text {. }\end{array}$ https://revistas.unimagdalena.edu.co/index.php/duazary/article/view/173

Rico-Hernández, C. (2016). Análisis de los estilos de enseñanza y aprendizaje aplicados en lengua castellana en docentes y estudiantes de la básica secundaria de la institución educativa San Francisco de la zona sur del municipio de Ibagué. [Tesis maestría en educación, Universidad Del Tolima]. Repositorio UT. http://repository.ut.edu.co/bitstream/001/1667/1/APROBADO\%20CAMILA\%20RIC O\%20HERN\%C3\%81NDEZ.pdf 
Rivera, L., Pirro, M., Vargas J., Bollet, F., y Flores, LE. (2017). Estilos de aprendizaje y de pensamiento en estudiantes de las diversas escuelas académico profesionales de la Universidad Privada De Pucallpa - Ucayali 2014. Revista de Investigación Científica - Pucallpa, 2(4), 5-8. https://doi.org/10.37292/riccva.v2i04.76

Rodríguez-Cepeda, R. (2016). Aprendizaje de conceptos químicos: una visión desde los trabajos prácticos y los estilos de aprendizaje. Rev.investig. desarro.innov, 7(1), 6376. https://doi.org/10.19053/20278306.v7.n1.2016.4403631

Saavedra, C., y Parra, J. (2014). Una mirada a los estilos de aprendizaje de los estudiantes de la UPTC desde la noción de native digital. Revista Academia y Virtualidad, 7(2), 41-5. https://revistas.unimilitar.edu.co/index.php/ravi/article/view/317

Salas-Cabrera, J. (2014). Estilos de aprendizaje en estudiantes de la Escuela de Ciencias del Movimiento Humano y Calidad de Vida, Universidad Nacional, Costa Rica. Revista Electrónica Educare, 18(3), 159-171. https://www.scielo.sa.cr/pdf/ree/v18n3/a09v18n3.pdf

Sánchez, J., y Alejos, B. (2019).Estilos de aprendizaje de estudiantes universitarios de ciencias biológicas. Journal of Learning Styles. 11(22), 1-21. http://revistaestilosdeaprendizaje.com/article/view/1210

Soriano, G. (2010). La Teoría de Piaget sobre el lenguaje y pensamiento del niño. https://piensoergoescribo.wordpress.com/2010/03/18/la-teoria-de-piaget-sobre-ellenguaje-y-pensamiento-del-nino/

Tobin, K. G., y Capie, W. (1981). The development and validation of a Group Test of Logical Thinking. Educational and Psychological Measurement, (41), 413-423.

Universidad Metropolitana. (2015). Bienestar universitario metropolitanade Barranquilla, Colombia. http://www.unimetro.edu.co/

Vidal, P., Canós, L., Santandreu, C., Guijarro, E. (2018). Influencia del estilo de aprendizaje del docente en el aula. INNODOCT. (14th-16th) 799-808. http://dx.doi.org/10.4995/INN2018.2018.8860

\section{Conflicto de intereses:}

Ninguno. 